\title{
A Volume Comparison Estimate with Radially Symmetric Ricci Curvature Lower Bound and Its Applications
}

\author{
Zisheng $\mathrm{Hu},{ }^{1}$ Yadong $\mathrm{Jin}{ }^{2}$ and Senlin $\mathrm{Xu}^{3}$ \\ ${ }^{1}$ School of Mathematics and Computational Science, Shenzhen University, Shenzhen, \\ Guangdong 518060, China \\ 2 School of Mathematics and Physics, Jiangsu Teachers University of Technology, Changzhou, \\ Jiangsu 213001, China \\ ${ }^{3}$ Department of Mathematics, University of Science and Technology of China, Hefei, Anhui 230026, China \\ Correspondence should be addressed to Zisheng Hu, zshu@szu.edu.cn
}

Received 3 July 2009; Revised 16 October 2009; Accepted 17 February 2010

Academic Editor: Mircea-Eugen Craioveanu

Copyright (C) 2010 Zisheng Hu et al. This is an open access article distributed under the Creative Commons Attribution License, which permits unrestricted use, distribution, and reproduction in any medium, provided the original work is properly cited.

We extend the classical Bishop-Gromov volume comparison from constant Ricci curvature lower bound to radially symmetric Ricci curvature lower bound, and apply it to investigate the volume growth, total Betti number, and finite topological type of manifolds with nonasymptotically almost nonnegative Ricci curvature.

\section{Introduction}

In comparison geometry of Ricci curvature, the classical Bishop-Gromov volume comparison has many applications, such as at least the linear volume growth of complete noncompact Riemannian manifolds with nonnegative Ricci curvature (see [1]), the upper bound of total Betti number (growth) of Riemannian manifolds (see [2-4]), and the finite topological type of complete noncompact Riemannian manifolds with nonnegative Ricci curvature or quadratic Ricci curvature decay (see $[3,5,6])$.

In [7], Lott and Shen establish a volume comparison estimate with quadratic Ricci curvature decay, and apply it to investigate the finite topological type of complete noncompact Riemannian manifolds with quadratic Ricci curvature decay, which generalizes a related result by Sha and Shen in [6].

In [8], we apply the volume comparison with asymptotically nonnegative Ricci curvature to investigate the corresponding topological results for manifolds with asymptotically nonnegative Ricci curvature. 
In this paper, we will extend the classical Bishop-Gromov volume comparison from constant Ricci curvature lower bound to general radially symmetric Ricci curvature lower bound, and apply it to investigate the volume growth, total Betti number and finite topological type of manifolds with non-asymptotically almost nonnegative Ricci curvature. (See Definitions 1.1 and 1.2 below for the notions of radially symmetric Ricci curvature lower bound, asymptotically almost nonnegative Ricci curvature, and non-asymptotically almost nonnegative Ricci curvature, resp.)

Note that quadratic Ricci curvature decay is non-asymptotically almost nonnegative Ricci curvature, so our result is a generalization of the corresponding result of Lott and Shen in [7] mentioned above. (See Theorem 1.7.)

Definition 1.1. Let $M$ be a complete $n$-Riemannian manifold $(n \geq 2), p \in M$, and $l:=$ $\sup \{d(p, x) \mid x \in M\} . M$ has a radially symmetric Ricci curvature lower bound, $k$ at the point $p$ if there exists a continuous function $k:[0, l) \rightarrow R$ such that, for any tangent vector $v \in T_{x} M$ radial from the point $p$,

$$
\operatorname{Ric}(v) \geq(n-1) k(d(p, x)) .
$$

One can refer to [9] for generalized space forms with radially symmetric curvature and the notion of tangent vector radial from a point.

Definition 1.2. Let $M$ a complete noncompact $n$-Riemannian manifold $(n \geq 2), p \in M, k$ : $[0, \infty) \rightarrow R$ be a continuous positive function, and $\lim _{t \rightarrow \infty} k(t)=0$.

(i) $M$ has almost nonnegative Ricci curvature if $\operatorname{Ric}(x) \geq(n-1) k(d(p, x))$.

Furthermore,

(ii) $M$ has asymptotically nonnegative Ricci curvature if $\operatorname{Ric}(x) \geq(n-1) k(d(p, x))$ and $C(k):=\int_{0}^{\infty} t k(t) d t<\infty$.

(iii) $M$ has non-asymptotically almost nonnegative Ricci curvature if $\operatorname{Ric}(x) \geq(n-$ 1) $k(d(p, x))$ and $C(k):=\int_{0}^{\infty} t k(t) d t=\infty$.

The following is a volume comparison estimate for manifolds with general radially symmetric Ricci curvature lower bound, which is a generalization of that for manifolds with asymptotically nonnegative Ricci curvature and quadratic Ricci curvature decay by Zhu in [10] and Lott and Shen in [7], respectively.

Theorem 1.3. Let $M$ be a complete $n$-Riemannian manifold $(n \geq 2)$ with a radially symmetric Ricci curvature lower bound $k:[0, l) \rightarrow R$ at the point $p \in M$, and let $r \leq R, s \leq S, r \leq s, R \leq S$, $\Gamma$ be a measurable subset of the unit sphere in the tangent space $T_{p} M$ :

$$
A_{r, R}^{\Gamma}(p):=\{x \in M \mid r \leq d(p, x) \leq R, \dot{\gamma}(0) \in \Gamma \text { for any minimal geodesic } \gamma \text { from } p \text { to } x\}
$$


Then

$$
\frac{\operatorname{vol}\left(A_{s, S}^{\Gamma}(p)\right)}{\operatorname{vol}\left(A_{r, R}^{\Gamma}(p)\right)} \leq \frac{\int_{s}^{S} y^{n-1}(t) d t}{\int_{r}^{R} y^{n-1}(t) d t}
$$

where $y(t)$ is the unique solution of one of the following two equations:

$$
\begin{gathered}
y^{\prime \prime}-k(t) y=0, \\
y(0)=0, \quad y^{\prime}(0)=1, \\
y^{\prime \prime}-k(t) y=0, \\
y(0)=0, \quad y^{\prime}(0)=1, \\
y>0 \quad \text { on }(0, l) .
\end{gathered}
$$

In particular, (1) if $y(t)$ is the unique solution of (1.4), then

$$
\operatorname{vol}\left(A_{s, S}^{\Gamma}(p)\right) \leq \operatorname{vol}(\Gamma) \int_{S}^{S} y^{n-1}(t) d t
$$

if $y(t)$ is the unique solution of (1.5), then

$$
\operatorname{vol}\left(A_{s, S}^{\Gamma}(p)\right) \leq \frac{\operatorname{vol}\left(A_{s, S}^{\Gamma}(p)\right)}{y^{n-1}(s)} \int_{s}^{S} y^{n-1}(t) d t
$$

(2) If there exist constants $C_{1}, C_{2}, K, L>0(0 \leq L-K<1 /(n-1))$ such that the unique solution of (1.4) or (1.5) satisfies $C_{1} t^{K} \leq y(t) \leq C_{2} t^{L}$, then one has a constant $C(n, k)>0$ depending only on $n$ and $k$ such that

$$
\operatorname{vol}\left(B_{p}(r+1)-B_{p}(r-1)\right) \leq C(n, k) \frac{\operatorname{vol}\left(B_{p}(r-1)\right)}{(r-1)^{1-(n-1)(L-K)}}
$$

Remark 1.4. The condition $y>0$ on $(0, l)$ in $(1.5)$ constitutes an extra assumption imposed on the unique solution $y$ of (1.4). In Theorem 1.3 , we do not require that the radially symmetric Ricci curvature lower bound $k:[0, l) \rightarrow R$ corresponds to the generalized space forms with radially symmetric curvature lower bound. Our purpose is to establish a volume comparison estimate effectively.

Applying the generalized volume comparison estimate, we can now investigate the volume growth, total Betti number, and finite topological type of manifolds with nonasymptotically almost nonnegative Ricci curvature.

Theorem 1.5. Let $M$ be a complete $n$-Riemannian manifold $(n \geq 2)$ with non-asymptotically almost nonnegative Ricci curvature $k:[0, l) \rightarrow R$ at the point $p \in M$, and let $M$ be noncollapsing, that 
is, $\inf _{x \in M} \operatorname{vol}\left(B_{x}(1)\right) \geq v>0$. If there exist constants $C_{1}, C_{2}, K, L>0(0 \leq L-K<1 /(n-1))$ such that, the unique solution of (1.4) or (1.5) satisfies $C_{1} t^{K} \leq y(t) \leq C_{2} t^{L}$, then one has a constant $C(n, k)>0$ depending only on $n$ and $k$ such that for $r>1$,

$$
\operatorname{vol}\left(B_{p}(r)\right) \geq C(n, k, v) r^{1-(n-1)(L-K)} .
$$

Theorem 1.6. Let $M$ be a complete $n$-Riemannian manifold $(n \geq 2)$ with non-asymptotically almost nonnegative Ricci curvature $k:[0, l) \rightarrow R$ at the point $p \in M$, and $M$ has weakly bounded geometry, that is, $\sec (M) \geq-1$, and $\inf _{x \in M} \operatorname{vol}\left(B_{x}\left(7^{-n-1} / 2\right)\right) \geq v>0$.

(1) If there exist constants $C, L>0$ such that the unique solution of (1.4) satisfies $y(t) \leq C_{2} t^{L}$, then one has a constant $C(n, k, v)>0$ depending only on $n, k$, and $v$ such that, for $r>0$,

$$
\sum_{i=0}^{n} b_{i}(p, r) \leq C(n, k, v)(1+r)^{\left(n^{2}-1\right) L+n+1} .
$$

(2) If there exist constants $C_{1}, C_{2}, K, L>0$ such that the unique solution of (1.5) satisfies $C_{1} t^{K} \leq y(t) \leq C_{2} t^{L}$, then one has a constant $C\left(n, k, v, \operatorname{vol}\left(S_{p}(1)\right), \operatorname{vol}\left(B_{p}(1)\right)\right)>0$ depending only on $n, k, v, \operatorname{vol}\left(S_{p}(1)\right)$, and $\operatorname{vol}\left(B_{p}(1)\right)$ such that, for $r>1$,

$$
\sum_{i=0}^{n} b_{i}(p, r) \leq C\left(n, C, v, \operatorname{vol}\left(S_{p}(1)\right), \operatorname{vol}\left(B_{p}(1)\right)\right)(1+r)^{\left(n^{2}-1\right) L+n+1} .
$$

Theorem 1.7. Let $M$ be a complete $n$-Riemannian manifold $(n \geq 2)$ with non-asymptotically almost nonnegative Ricci curvature $k:[0, l) \rightarrow R$ at the point $p \in M, \sec (x) \geq-\left(C / d(p, x)^{\alpha}\right)$ where $C>0,0 \leq \alpha \leq 2$, and let $M$ be non-collapsing, that is, $\inf _{x \in M} \operatorname{vol}\left(B_{x}(1)\right) \geq v>0$. If there exist constants $C_{1}, C_{2}, K, L>0(0 \leq L-K<(1 /(n-1))$ such that the unique solution of (1.4) or (1.5) satisfies $C_{1} t^{K} \leq y(t) \leq C_{2} t^{L}$, then $M$ is of finite topological type with the additional assumption that

$$
\limsup _{r \rightarrow \infty} \frac{\operatorname{vol}\left(B_{p}(r)\right)}{r^{1+(\alpha / 2)-(n-1)(L-K)}}<C(n, k, C, \alpha, v)
$$

for some constant $\widetilde{C}(n, k, C, \alpha, v)>0$ depending only on $n, k, C, \alpha$, and $v$.

\section{A Volume Comparison Estimate with Radially Symmetric Ricci Curvature Lower Bound}

Proof of Theorem 1.3. Choose polar coordinate $(r, \theta)$ at $p$. Define the function $J(r, \theta)$ by the formula

$$
d v_{M}=J^{n-1} d r d \theta .
$$

Then

$$
\operatorname{vol}\left(A_{s, S}^{\Gamma}(p)\right)=\int_{\Gamma} d \theta \int_{\min \{s, \operatorname{cut}(\theta)\}}^{\min \{S, \operatorname{cut}(\theta)\}} J^{n-1} d r,
$$


where $\operatorname{cut}(\theta)$ is the distance from $p$ to the cut point in direction $\theta$. It is well known (e.g., [11]) that $J$ satisfies the following:

$$
\begin{gathered}
J^{\prime \prime}-k J \leq 0, \quad 0 \leq t \leq \operatorname{cut}(\theta), \\
J(0)=0, \quad J^{\prime}(0)=1 .
\end{gathered}
$$

Let $y(t)$ be the unique solution of one of (1.4) and (1.5) (Note that, by the uniqueness of the solution of ordinary differential equation, the solution of (1.4) always exists.).

Then in the interval of $y>0, J^{\prime \prime} y-y^{\prime \prime} J \leq 0$, that is, $\left(J^{\prime} y-y^{\prime} J\right)^{\prime} \leq 0$. By the initial condition of $J$ and $y, J^{\prime} y-y^{\prime} J \leq 0$. Thus, when $y>0$,

$$
\left(\frac{J}{y}\right)^{\prime}=\frac{1}{y^{2}}\left(J^{\prime} y-y^{\prime} J\right) \leq 0
$$

This shows that $J / y$ is nonincreasing in the interval of $y>0$.

Note that in the interval of $J>0$ we must have $y>0$. Thus it suffices to consider that $y(t)$ is the unique solution of (1.4).

Otherwise, suppose that $t_{0}>0$ is the first point such that $y>0$ in $\left(0, t_{0}\right), y\left(t_{0}\right)=0$, and $J>0$ in $\left(0, t_{0}\right]$. By $J(0)=y(0)=0, J^{\prime}(0)=y^{\prime}(0)=1, J / y$ is non-increasing in $\left(0, t_{0}\right)$ :

$$
\begin{gathered}
\frac{J}{y}(0):=\lim _{t \rightarrow 0} \frac{J(t)}{y(t)}=\lim _{t \rightarrow 0} \frac{J^{\prime}(t)}{y^{\prime}(t)}=1, \\
\frac{J}{y}(t) \leq \frac{J}{y}(0)=1, \quad t \in\left(0, t_{0}\right), \\
J(t) \leq y(t), \quad t \in\left(0, t_{0}\right) .
\end{gathered}
$$

Let $t \rightarrow t_{0}$, then $J\left(t_{0}\right) \leq y\left(t_{0}\right)=0$. This is a contradiction.

Thus consider the following lemma.

Lemma 2.1 (see [12]). Let $f, g$ be positive functions on $[0,+\infty)$; if $f / g$ is nonincreasing, then for all $R>r>0, S>s>0, s>r, S>R$, one has

$$
\frac{\int_{S}^{S} f(t) d t}{\int_{r}^{R} f(t) d t} \leq \frac{\int_{s}^{S} g(t) d t}{\int_{r}^{R} g(t) d t} .
$$


We have

$$
\begin{aligned}
\frac{\int_{\min \{r, \operatorname{cut}(\theta)\}}^{\min \{S, \operatorname{cut}(\theta)\}} J^{n-1}(t, \theta) d t}{\int_{\min \{r, \operatorname{cut}(\theta)\}}^{\min \{R, \operatorname{cut}(\theta)} J^{n-1}(t, \theta) d t} & \leq \frac{\int_{\min \{r, \operatorname{cut}(\theta)\}}^{\min \{S, \operatorname{cut}(\theta)\}} y^{n-1}(t) d t}{\int_{\min \{r, \operatorname{cut}(\theta)\}}^{\min \{R, \operatorname{cut}(\theta)} y^{n-1}(t) d t} \\
& \leq \frac{\int_{r}^{\min \{S, \operatorname{cut}(\theta)\}} y^{n-1}(t) d t}{\int_{r}^{\min \{R, \operatorname{cut}(\theta)\}} y^{n-1}(t) d t} \\
& \left.\leq \frac{\int_{r}^{S} y^{n-1}(t) d t}{\int_{r}^{R} y^{n-1}(t) d t}, \quad \text { (because } R<S\right),
\end{aligned}
$$

where the last equality is due to

$$
\frac{\int_{r}^{\min \{S, \operatorname{cut}(\theta)\}} y^{n-1}(t) d t}{\int_{r}^{\min \{R, \operatorname{cut}(\theta)\}} y^{n-1}(t) d t}= \begin{cases}\frac{\int_{r}^{\operatorname{cut}(\theta)} y^{n-1}(t) d t}{\int_{r}^{\operatorname{cut}(\theta)} y^{n-1}(t) d t}=1, & \text { when } \operatorname{cut}(\theta) \leq R \leq S, \\ \frac{\int_{r}^{\operatorname{cut}(\theta)} y^{n-1}(t) d t}{\int^{n-1}(t) d t}, & \text { when } R \leq \operatorname{cut}(\theta) \leq S, \\ \frac{\int_{r}^{S} y^{n-1}(t) d t}{\int_{r}^{R} y^{n-1}(t) d t}, & \text { when } R \leq S \leq \operatorname{cut}(\theta) .\end{cases}
$$

Then by integration on $\Gamma$, we have

$$
\frac{\operatorname{vol}\left(A_{r, S}^{\Gamma}(p)\right)}{\operatorname{vol}\left(A_{r, R}^{\Gamma}(p)\right)} \leq \frac{\int_{r}^{S} y^{n-1}(t) d t}{\int_{r}^{R} y^{n-1}(t) d t} .
$$

Similarly,

$$
\frac{\operatorname{vol}\left(A_{s, S}^{\Gamma}(p)\right)}{\operatorname{vol}\left(A_{r, R}^{\Gamma}(p)\right)} \leq \frac{\int_{s}^{S} y^{n-1}(t) d t}{\int_{r}^{R} y^{n-1}(t) d t} .
$$


In particular, (1)

$$
\begin{aligned}
\operatorname{vol}\left(A_{s, S}^{\Gamma}(p)\right) & \leq \frac{\operatorname{vol}\left(A_{r, R}^{\Gamma}(p)\right)}{\int_{r}^{R} y^{n-1}(t) d t} \int_{S}^{S} y^{n-1}(t) d t \\
& =\frac{\int_{\Gamma} \int_{\min \{r, \operatorname{cut}(\theta)\}}^{\min \{R, \operatorname{cut}(\theta)} J^{n-1}(t, \theta) d t d \theta}{\int_{r}^{R} y^{n-1}(t) d t} \int_{S}^{S} y^{n-1}(t) d t \\
& =\int_{\Gamma} \frac{\int_{\min \{r, \operatorname{cut}(\theta)\}}^{\min \{R, \operatorname{cut}(\theta)\}} J^{n-1}(t, \theta) d t}{\int_{r}^{R} y^{n-1}(t) d t} d \theta \int_{S}^{S} y^{n-1}(t) d t .
\end{aligned}
$$

Let $R \rightarrow r$, then

$$
\operatorname{vol}\left(A_{s, S}^{\Gamma}(p)\right) \leq \int_{\Gamma} \frac{J^{n-1}(r, \theta)}{y^{n-1}(r)} d \theta \int_{S}^{S} y^{n-1}(t) d t
$$

When $y(t)$ is the unique solution of (1.4), let $r \rightarrow 0$; by $J(0)=y(0)=0, J^{\prime}(0)=y^{\prime}(0)=$ 1 ; then we have

$$
\operatorname{vol}\left(A_{s, S}^{\Gamma}(p)\right) \leq \operatorname{vol}(\Gamma) \int_{S}^{S} y^{n-1}(t) d t
$$

When $y(t)$ is the unique solution of (1.5), let $r=s$, we have

$$
\begin{aligned}
\operatorname{vol}\left(A_{s, S}^{\Gamma}(p)\right) & \leq \int_{\Gamma} \frac{J^{n-1}(s, \theta)}{y^{n-1}(s)} d \theta \int_{S}^{S} y^{n-1}(t) d t \\
& =\frac{\operatorname{vol}\left(A_{s, S}^{\Gamma}(p)\right)}{y^{n-1}(s)} \int_{S}^{S} y^{n-1}(t) d t .
\end{aligned}
$$


(2) Choose $\Gamma=S_{p}^{n-1}$; for $r \geq 3$, an easy computation shows that

$$
\begin{aligned}
& \frac{\operatorname{vol}\left(B_{p}(r+1)-B_{p}(r-1)\right)}{\operatorname{vol}\left(B_{p}(r-1)-B_{p}(1)\right)} \\
& \leq \frac{\int_{r-1}^{r+1} y^{n-1}(t) d t}{\int_{1}^{r-1} y^{n-1}(t) d t} \\
& \leq \frac{\int_{r-1}^{r+1}\left(C_{2} t^{L}\right)^{n-1} d t}{\int_{1}^{r-1}\left(C_{1} t^{K}\right)^{n-1} d t} \\
& =\frac{C_{2}^{n-1}((n-1) K+1)}{C_{1}^{n-1}((n-1) L+1)} \cdot \frac{(r+1)^{(n-1) L+1}-(r-1)^{(n-1) L+1}}{(r-1)^{(n-1) K+1}-1} \\
& \leq \frac{2 C_{2}^{n-1}((n-1) K+1)}{C_{1}^{n-1}((n-1) L+1)} \cdot \frac{(r+1)^{(n-1) L+1}-(r-1)^{(n-1) L+1}}{(r-1)^{(n-1) K+1}} \\
& =C\left(n, C_{1}, C_{2}, K, L\right)\left(\left(1+\frac{2}{r-1}\right)^{(n-1) K+1} \cdot(r+1)^{(n-1)(L-K)}-(r-1)^{(n-1)(L-K)}\right) \\
& \leq C\left(n, C_{1}, C_{2}, K, L\right)\left(\left(1+\frac{C(n, K)}{r-1}\right) \cdot(r+1)^{(n-1)(L-K)}-(r-1)^{(n-1)(L-K)}\right) \\
& =C\left(n, C_{1}, C_{2}, K, L\right) \cdot(r-1)^{(n-1)(L-K)} \cdot\left(\left(1+\frac{C(n, K)}{r-1}\right) \cdot\left(\frac{r+1}{r-1}\right)^{(n-1)(L-K)}-1\right) \\
& \leq C\left(n, C_{1}, C_{2}, K, L\right) \cdot(r-1)^{(n-1)(L-K)} \cdot\left(\left(1+\frac{C(n, K)}{r-1}\right) \cdot\left(1+\frac{C(n, K, L)}{r-1}\right)-1\right) \\
& =C\left(n, C_{1}, C_{2}, K, L\right) \cdot(r-1)^{(n-1)(L-K)} \cdot\left(\frac{C(n, K)+C(n, K, L)}{r-1}+\frac{C(n, K) \cdot C(n, K, L)}{(r-1)^{2}}\right) \\
& \leq C\left(n, C_{1}, C_{2}, K, L\right) \frac{1}{(r-1)^{1-(n-1)(L-K)}} .
\end{aligned}
$$

\section{Proof of Theorem 1.5}

Proof of Theorem 1.5. Note that for $r \geq 3$ there exists a point $q \in S_{p}(r)$ such that $\left(B_{p}(r+1)-\right.$ $\left.B_{p}(r-1)\right) \supseteq B_{q}(1)$; thus

$$
\operatorname{vol}\left(B_{p}(r+1)-B_{p}(r-1)\right) \geq \operatorname{vol} B_{q}(1) .
$$

And since $M$ does not collapse at infinity, that is, $\inf _{x \in M} \operatorname{vol}\left(B_{x}(1)\right) \geq v>0$, for $r \geq 3$, we have

$$
\operatorname{vol}\left(B_{p}(r+1)-B_{p}(r-1)\right) \geq v
$$


Thus, for $r \geq 3$, by Theorem 1.3(2), there is some constant $C(n, k, v)$ such that $\operatorname{vol}\left(B_{p}(r)\right) \geq$ $C(n, k, v) r^{1-(n-1)(K-L)}$. And note that for $1<r \leq 3$,

$$
B_{p}(r) \supseteq B_{p}(1)
$$

Theorem 1.5 is obtained.

\section{Proof of Theorem 1.6}

First let us recall Gromov's theorems [2]; one can refer to [13] for the details.

Theorem 4.1 (see [2]). Let $M$ be an $n$-dimensional complete Riemannian manifold with sectional curvature $K \geq-1$. Then there is a constant $C(n)>1$ depending only on $n$ such that, for any $0<\epsilon<1$ and any bounded subset $X \subset M$,

$$
\sum_{k=0}^{n} b_{k}\left(X, T_{\epsilon} X\right) \leq\left(1+\operatorname{diam}(X) \epsilon^{-1}\right)^{n} C(n)^{1+\operatorname{dia}(X)}
$$

where $T_{\epsilon} X$ denotes the $\epsilon$-neighborhood of $X$ in $M$.

Theorem 4.2 (see [2]). Let $M$ be an n-dimensional complete Riemannian manifold and let $p \in M$. For any fixed numbers $r>0$ and $r_{0} \leq 7^{-n-1}$, let $B_{j}^{0}:=B\left(p_{j}, r_{0}\right), j=1, \ldots, N$, be a ball covering of $B(p, r)$ with $p_{j} \in B(p, r)$. Let $B_{j}^{k}:=7^{k} B_{j}^{0}:=B\left(p_{j}, 7^{k} r_{0}\right), k=0, \ldots, n+1$. Then

$$
\sum_{i=0}^{n} b_{i}(B(p, r), B(p, r+1)) \leq(e-1) N t^{n} \sup \left\{\sum_{i=0}^{n} b_{i}\left(B_{j}^{k}, 5 B_{j}^{k}\right): 0 \leq k \leq n, 1 \leq j \leq N\right\},
$$

where $t$ is the smallest number such that, each ball $B_{j}^{n}$ intersects at most tother balls $B_{j^{\prime}}^{n}$.

Proof of Theorem 1.6. By Theorem 4.1, there is a constant $C(n)$ depending only on $n$ such that for all balls $B(x, r)$ with radius $r \leq 1$ in $M$,

$$
\sum_{i=0}^{n} b_{i}(B(x, r), B(x, 5 r)) \leq C_{1}(n)
$$

Take $r_{0}=7^{-n-1}$, and let $B\left(p_{j},(1 / 2) r_{0}\right), j=1, \ldots, N$, be a maximal set of disjoint balls with $p_{j} \in B(p, r)$, and let $B_{j}^{k}, j=1, \ldots, N, k=0, \ldots, n+1$, be the same as in Theorem 4.2. Then $B_{j}^{0}, j=1, \ldots, N$, is a covering of $B(p, r)$. And let $t, N$ be the same as in Theorem 4.2. 
If there exist constants $C, L>0$ such that the unique solution of (1.4) satisfies $y(t) \leq$ $C_{2} t^{L}$, choosing $\Gamma=S_{p}^{n-1}, s=0$ in Theorem 1.3(1), then, for $S \geq 0$,

$$
\begin{aligned}
\operatorname{vol}\left(B_{p}(S)\right) & \leq \operatorname{vol}\left(S_{1}^{n-1}\right) \int_{0}^{S}\left(C_{2} t^{L}\right)^{n-1} d t \\
& =\operatorname{vol}\left(S_{1}^{n-1}\right) \frac{C_{2}}{(n-1) L+1} S^{(n-1) L+1} \\
& =: C(n, k) S^{(n-1) L+1} .
\end{aligned}
$$

Then by the assumption that $\inf _{x \in M} \operatorname{vol}\left(B_{x}\left(7^{-n-1} / 2\right)\right) \geq v>0$,

$$
\begin{aligned}
N & \leq \frac{\operatorname{vol}\left(B_{x}\left(r+\left(r_{0} / 2\right)\right)\right)}{\min _{j} \operatorname{vol}\left(B_{p_{j}}\left(r_{0} / 2\right)\right)} \\
& \leq \frac{C(n, k)\left(r+\left(r_{0} / 2\right)\right)^{(n-1) L+1}}{v} \\
& \leq \frac{C(n, k)(r+1)^{(n-1) L+1}}{v} \\
t & \leq \frac{\operatorname{vol}\left(B_{p_{j}}\left((2 / 7)+\left(r_{0} / 2\right)\right)\right)}{\min _{j^{\prime}} \operatorname{vol}\left(B_{p_{j^{\prime}}}\left(r_{0} / 2\right)\right)} \\
& \leq \frac{\operatorname{vol}\left(B_{p}\left(r+(2 / 7)+\left(r_{0} / 2\right)\right)\right)}{v} \\
& \leq \frac{C(n, k)\left(r+(2 / 7)+\left(r_{0} / 2\right)\right)^{(n-1) L+1}}{v} \\
& \leq \frac{C(n, k)(r+1)^{(n-1) L+1}}{v} .
\end{aligned}
$$

Since each ball $B_{j}^{k}$ has radius $\leq 1$, it follows from (4.3) and Theorem 4.2 that

$$
\begin{aligned}
\sum_{i=0}^{n} b_{i}\left(B_{p}(r), M\right) & \leq \sum_{i=0}^{n} b_{i}\left(B_{p}(r), B_{p}(r+1)\right) \\
& \leq(e-1)\left(\frac{C(n, k)(r+1)^{(n-1) L+1}}{v}\right)^{n+1} C(n) \\
& =: C(n, k, v)(1+r)^{((n-1) L+1)(n+1)} .
\end{aligned}
$$


If there exist constants $C_{1}, C_{2}, K, L>0$ such that the unique solution of (1.5) satisfies $C_{1} t^{K} \leq y(t) \leq C_{2} t^{L}$, choosing $\Gamma=S_{p}^{n-1}, s=1$ in Theorem 1.3(1), then, for $S \geq 1$,

$$
\begin{aligned}
\operatorname{vol}\left(B_{p}(S)-B_{p}(1)\right) & \leq \frac{\operatorname{vol}\left(S_{p}(1)\right)}{y^{n-1}(1)} \int_{1}^{S} y^{n-1}(t) d t \\
& \leq \frac{\operatorname{vol}\left(S_{p}(1)\right)}{C_{1}} \int_{1}^{S}\left(C_{2} t^{L}\right)^{n-1} d t \\
& \leq \frac{\operatorname{vol}\left(S_{p}(1)\right)}{C_{1}} \frac{C_{2}}{(n-1) L+1} S^{(n-1) L+1} \\
& =: C\left(n, k, \operatorname{vol}\left(S_{p}(1)\right)\right) S^{(n-1) L+1}, \\
\operatorname{vol}\left(B_{p}(S)\right) & \leq C\left(n, k, \operatorname{vol}\left(S_{p}(1)\right)\right) S^{(n-1) L+1}+\operatorname{vol}\left(B_{p}(1)\right) \\
& \leq C\left(n, k, \operatorname{vol}\left(S_{p}(1)\right), \operatorname{vol}\left(B_{p}(1)\right)\right) S^{(n-1) L+1}
\end{aligned}
$$

Similar to the above, there exists a constant $C\left(n, k, \operatorname{vol}\left(S_{p}(1)\right), \operatorname{vol}\left(B_{p}(1)\right)\right)>0$ such that

$$
\sum_{i=0}^{n} b_{i}\left(B_{p}(r), M\right) \leq C\left(n, k, \operatorname{vol}\left(S_{p}(1)\right), \operatorname{vol}\left(B_{p}(1)\right)\right)(1+r)^{((n-1) L+1)(n+1)}
$$

\section{Proof of Theorem 1.7}

We use critical point theory of the distance function to prove Theorem 1.7.

First of all, we recall some concepts (cf., e.g., $[3,7,14]$ ). Notice that the distance function $d_{p}(x):=d(p, x)$ is not a smooth function (on the cutlocus of $p$ ). Hence the critical points of $d_{p}$ are not defined in a usual sense. The notion of critical points of $d_{p}$ is introduced by Grove and Shiohama [15].

A point $x \in M$ is called a critical point of $d_{p}$ if for any unit vector $v \in T_{x} M$ there is a minimizing geodesic $\sigma$ from $x$ to $p$ such that $\angle\left(\sigma^{\prime}(0), v\right) \leq \pi / 2$.

For every $r$, the open set $M \backslash \overline{B(p, r)}$ contains only finitely many unbounded components, $U_{r}$. Each $U_{r}$ has finitely many boundary components, $\Sigma_{r} \subset \partial B(p, r)$. In particular, $\Sigma_{r}$ is a closed subset. Let us say that a connected component $\Sigma_{r}$ of $S(p, r)$ is good if it is part of the boundary of an unbounded component of $M \backslash \overline{B(p, r)}$ and there is a ray from $p$ passing through $\Sigma_{r}$.

Now we can introduce the following lemma.

Lemma 5.1 (see (Lemma 3.2, [7]); cf., also [14]). Suppose that there is a $r_{0}>0$ such that if $r>r_{0}$ then there is no critical point of $d_{p}$ on any good component $\Sigma_{r}$ of $S(p, r)$. Then $M$ has finite topological type.

Another concept is the diameter growth function $\Phi(p, r)$. 
Definition 5.2. The diameter growth function $\Phi(p, r)$ is defined by

$$
\Phi(p, r)=\sup _{\Sigma_{r}} \operatorname{diam}\left(\sum_{r}\right)
$$

where the supremum is taken over all good components $\Sigma_{t}$ of $S_{t}$ and the diameter is measured using the metric on $M$.

Proof of Theorem 1.7. (i) We first show that if a complete noncompact Riemannian manifold satisfies $K(x) \geq-C / d(p, x)^{\alpha}$, where $C>0,0 \leq \alpha \leq 2$, and the following diameter growth condition

$$
\limsup _{r \rightarrow \infty} \frac{\Phi(p, r)}{r^{\alpha / 2}}<\frac{\delta_{1}}{2}
$$

where

$$
\delta_{1}=\max _{0<\epsilon \leq 1 / 20}\left\{2 \epsilon-\frac{\operatorname{arc} \cosh \left(\cosh ^{2} 2^{\alpha} C^{1 / 2} \epsilon\right)}{2^{\alpha} C^{1 / 2}}>0\right\}
$$

then $M$ is of finite topological type.

As (i) in the proof of Theorem 1.1 in [8], choose a good connected component $\Sigma_{r}$ of $S(p, r)$, for any $x \in \Sigma_{r}$, and a ray $\gamma$ from $p$ passing through $\Sigma_{r}$, choose $q=\gamma(t)$ such that $t \geq 2 d(p, x)$, and suppose that $x$ is a critical point of $d_{p}$, then

$$
e_{p q}(x) \geq \delta_{1} d(p, x)^{\alpha / 2}
$$

On the other hand, by the triangle inequality,

$$
e_{p q}(x) \leq 2 \Phi(p, r)
$$

thus,

$$
\Phi(p, r) \geq \frac{\delta_{1}}{2} d(p, x)^{\alpha / 2}
$$

For $r$ large enough, by the assumption on the diameter growth, this is a contradiction.

Thus, there does not exist a critical point of $d(p, \cdot)$ on any good connected component. By Lemma 5.1, $M$ is of finite topological type.

(ii) Given that $r>0$, choose a good connected component $\Sigma_{r}$, of the boundary of an unbounded component of $M \backslash \overline{B(p, r)}$. For any $x, y \in \Sigma_{r}$, there is a continuous curve $c:[0, s] \rightarrow \Sigma_{r}$ from $x$ to $y$. Suppose that $d(x, y)>2$. Then there is a partition 
$0=t_{0}<t_{1}<\cdots<t_{k}=r$ such that $\left\{B\left(c\left(t_{i}\right), 1\right)\right\}_{i=0}^{k}$ are disjoint and $B\left(c\left(t_{i}\right), 2\right) \cap B\left(c\left(t_{i+1}\right), 2\right) \neq \emptyset$. Note that $B\left(c\left(t_{i}\right), 1\right) \subset B(p, r+1)-\overline{B(p, r-1)}$. Thus

$$
\begin{gathered}
(k+1) v \leq \sum_{i=0}^{k} \operatorname{vol}\left(B\left(c\left(t_{i}\right), 1\right)\right) \leq \operatorname{vol}(B(p, r+1)-\overline{B(p, r-1)}), \\
\operatorname{diam}\left(\sum_{r}\right) \leq \sum_{i=0}^{k-1} d\left(c\left(t_{i}\right), c\left(t_{i+1}\right)\right) \leq C(n, k, v) \operatorname{vol}\left(B_{p}(r+1)-\overline{B_{p}(r-1)}\right) .
\end{gathered}
$$

Then, by Theorem 1.3(2), there is a constant $\widetilde{C}(n, k, C, \alpha, v)$ such that if the volume growth satisfies

$$
\limsup _{r \rightarrow \infty} \frac{\operatorname{vol}\left(B_{p}(r)\right)}{r^{1+(\alpha / 2)-(n-1)(L-K)}}<\widetilde{C}(n, k, C, \alpha, v)
$$

the diameter growth satisfies

$$
\limsup _{r \rightarrow \infty} \frac{\Phi(p, r)}{r^{\alpha / 2}}<\frac{\delta_{1}}{2}
$$

Then by (i), Theorem 1.7 is obtained.

\section{Acknowledgment}

The authors would like to thank the referee for the comments and suggestions.

\section{References}

[1] S. T. Yau, "Some function-theoretic properties of complete Riemannian manifold and their applications to geometry," Indiana University Mathematics Journal, vol. 25, no. 7, pp. 659-670, 1976.

[2] M. Gromov, "Curvature, diameter and Betti numbers," Commentarii Mathematici Helvetici, vol. 56, no. 2, pp. 179-195, 1981.

[3] Z. Shen, "On complete manifolds of nonnegative $k$ th-Ricci curvature," Transactions of the American Mathematical Society, vol. 338, no. 1, pp. 289-310, 1993.

[4] Z. Shen and G. Wei, "Ricci curvature and Betti numbers," Journal of Geometric Analysis, vol. 7, no. 3, pp. 493-509, 1997.

[5] Z. Shen and G. Wei, "Volume growth and finite topological type," in Differential Geometry: Riemannian Geometry, vol. 54 of Proc. Sympos. Pure Math., pp. 539-549, American Mathematical Society, Providence, RI, USA, 1993.

[6] J. Sha and Z. Shen, "Complete manifolds with nonnegative Ricci curvature and quadratically nonnegatively curved infinity," American Journal of Mathematics, vol. 119, no. 6, pp. 1399-1404, 1997.

[7] J. Lott and Z. Shen, "Manifolds with quadratic curvature decay and slow volume growth," Annales Scientifiques de l'École Normale Supérieure, vol. 33, no. 2, pp. 275-290, 2000.

[8] Z. Hu and S. Xu, "Complete manifolds with asymptotically nonnegative Ricci curvature and weak bounded geometry," Archiv der Mathematik, vol. 88, no. 5, pp. 455-467, 2007.

[9] N. Katz and K. Kondo, "Generalized space forms," Transactions of the American Mathematical Society, vol. 354, no. 6, pp. 2279-2284, 2002.

[10] S. Zhu, "A volume comparison theorem for manifolds with asymptotically nonnegative curvature and its applications," American Journal of Mathematics, vol. 116, no. 3, pp. 669-682, 1994. 
[11] P. Petersen, Riemannian Geometry, vol. 171 of Graduate Texts in Mathematics, Springer, New York, NY, USA, 1998.

[12] S. Zhu, "The comparison geometry of Ricci curvature," in Comparison Geometry, vol. 30 of Math. Sci. Res. Inst. Publ., pp. 221-262, Cambridge University, Cambridge, UK, 1997.

[13] U. Abresch, "Lower curvature bounds, Toponogov's theorem, and bounded topology. II," Annales Scientifiques de l'École Normale Supérieure, vol. 20, no. 3, pp. 475-502, 1987.

[14] J. Cheeger, "Critical points of distance functions and applications to geometry," in Lecture Notes in Mathematics, vol. 1504, pp. 1-38, Springer, Heidelberg, Germany, 1991.

[15] K. Grove and K. Shiohama, "A generalized sphere theorem," Annals of Mathematics, vol. 106, no. 2, pp. 201-211, 1977. 


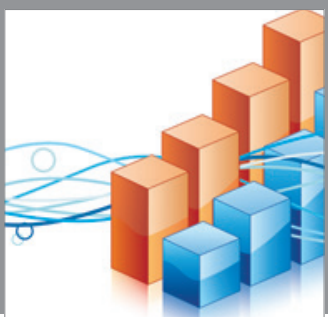

Advances in

Operations Research

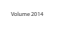

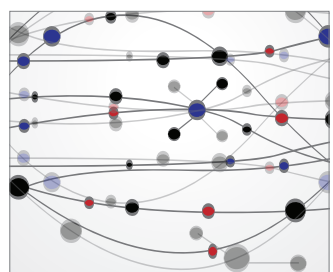

\section{The Scientific} World Journal
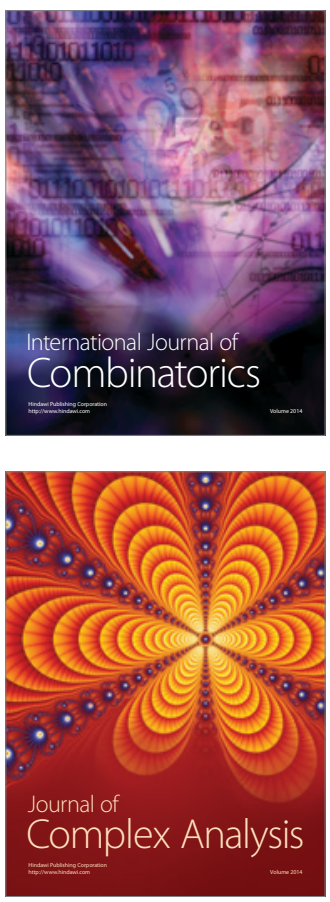

International Journal of

Mathematics and

Mathematical

Sciences
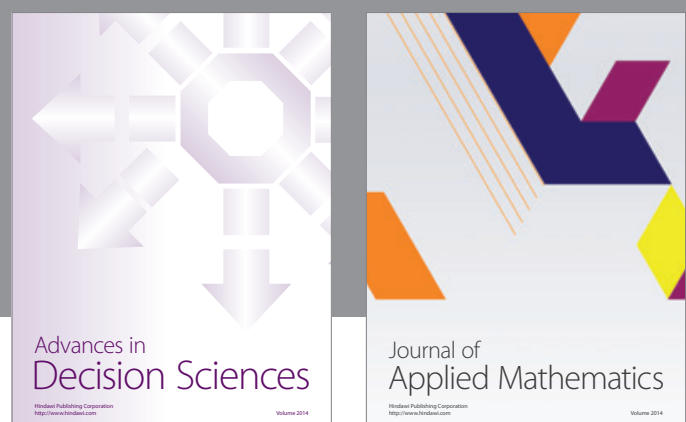

Journal of

Applied Mathematics
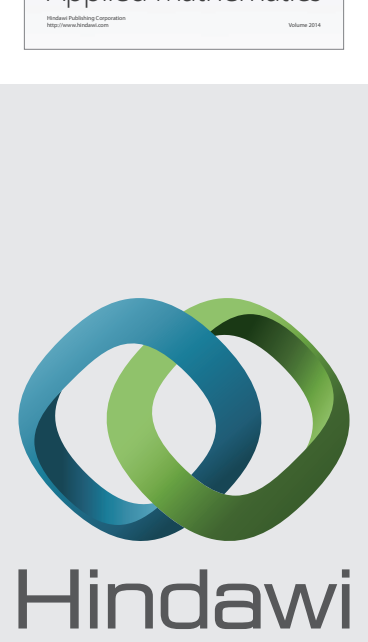

Submit your manuscripts at http://www.hindawi.com
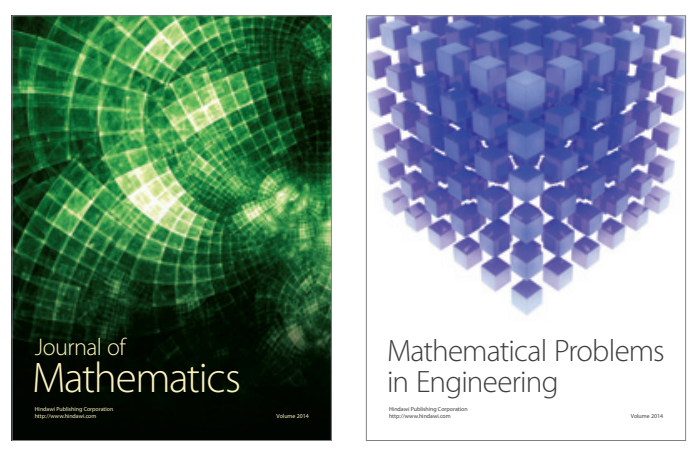

Mathematical Problems in Engineering
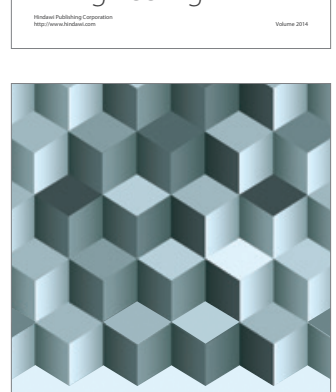

Journal of

Function Spaces
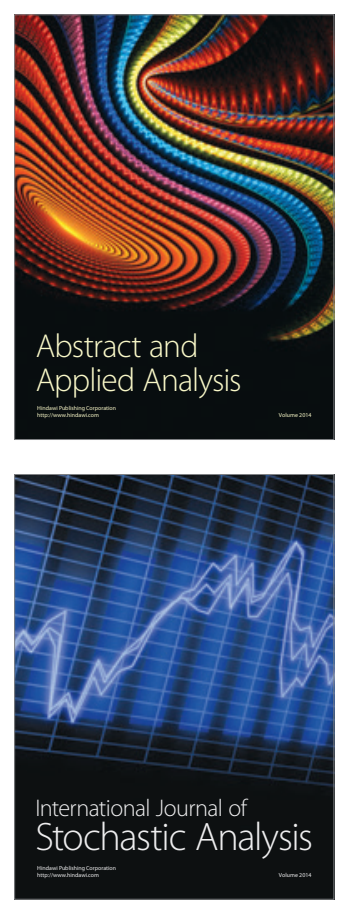

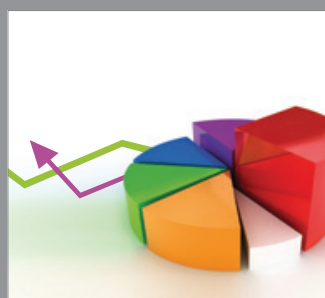

ournal of

Probability and Statistics

Promensencen
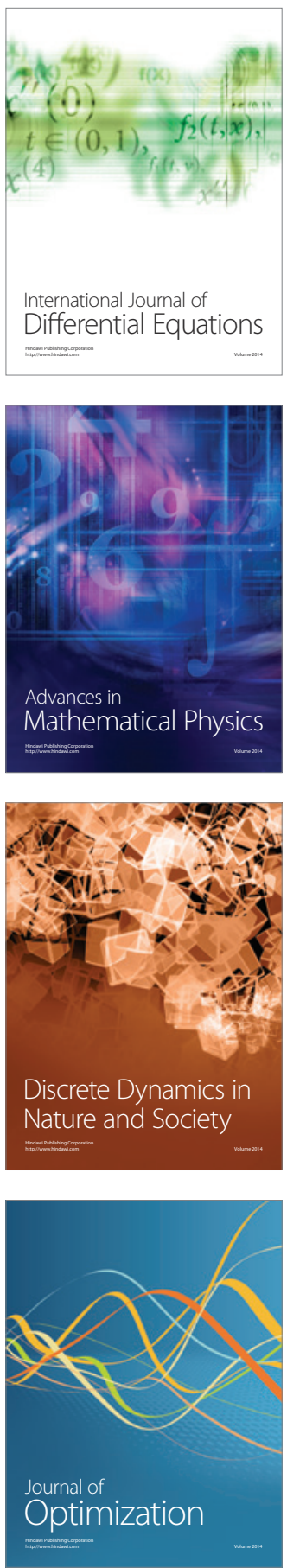